\title{
Surface Reflectance Characterization by Statistical Tools
}

\author{
Vlastimil Havran* \\ Czech Technical University \\ Faculty of Electrical Engineering
}
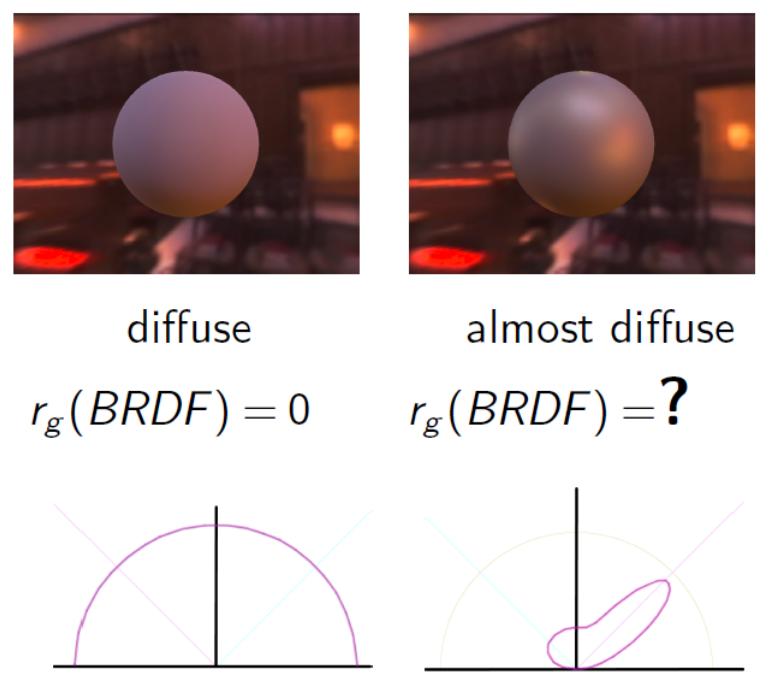

\author{
Mateu Sbert ${ }^{\dagger}$ \\ University of Girona \\ Graphics and Imaging Laboratory
}

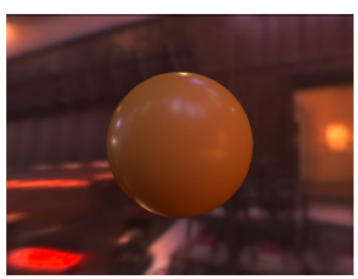

slightly glossy

$r_{g}(B R D F)=?$

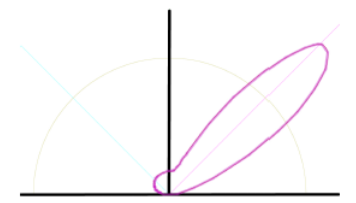

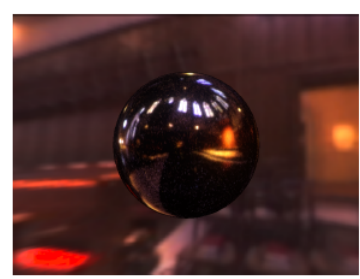

specular

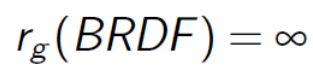

Figure 1: A statistical definition of glossiness of surface reflectance, named variant index of glossiness $r_{g}\left(f_{r}\left(x, \omega_{o}\right)\right)$ (abbreviated as VIG), for the rendering purposes is studied and proposed in this paper to extend the Heckbert's notation for diffuse, glossy, and specular BRDFs.

\section{Abstract}

The classification of surface reflectance functions as diffuse, specular, and glossy has been introduced by Heckbert more than two decades ago. Many rendering algorithms are dependent on such a classification, as different kinds of light transport will be handled by specialized methods, for example, caustics require specular bounce or refraction. As the surface reflectance models are more and more rich and descriptive including those based on measured data, it has not been possible to keep such a characterization simple. Each surface reflectance model is mostly handled separately, or alternatively, the rendering algorithm restricts itself to the use of some subset of reflectance models. We provide a general characterization for arbitrary surface reflectance representation by means of statistical tools. We demonstrate by rendered images using Matusik's BRDF data sets for two environment maps and two 3D objects (sphere and Utah teapot) that there is even a visible perceptual correspondence to the proposed surface reflectance characterization, when we use monochromatic surface reflectance and the albedo is normalized for rendering images to equalize perceived brightness. The proposed characterization is intended to be used to optimize rendering algorithms.

CR Categories: I.3.3 [Computer Graphics]: Three-Dimensional Graphics and Realism-Display Algorithms I.3.7 [Computer Graphics]: Three-Dimensional Graphics and Realism-Radiosity;

\section{Introduction}

The surface reflectance has been studied in many scientific fields including computer graphics, computer vision, optics, remote sensing, visual appearance etc. The formalization of surface reflectance known as bidirectional reflectance distribution function (BRDF) [Nicodemus et al. 1977] is in the core of the rendering equation [Kajiya 1986]. It is known long time that some classes of rendering algorithms are suited for some surface reflectance; i.e. the recursive ray tracing is efficient for mirror-like surfaces, while radiosity is efficient for diffuse surfaces. These limitations of rendering algorithms made the research oriented to general solutions that ideally would work independently on the surface reflectance used. In many research papers and applications the algorithms pose these limitations in form of exponent of Phong model or specular roughness of Ward model. Sometimes the limitations are specified in papers vaguely in terms as 'almost diffuse, moderately glossy', highly glossy etc. However, such specification of the surface reflectance characterization is crucial for both visual quality of images and performance of many rendering algorithms.

In our paper we propose a simple but powerful characterization of surface reflectance that can be used in rendering algorithms irrespective if they are biased, consistent, or unbiased. We provide the analysis using statistical tools on how the surface reflectance influ-

Keywords: bidirectional texture function, variance, diffuseness, glossiness

\footnotetext{
*e-mail:havran@fel.cvut.cz

†e-mail:sbert@ima.udg.edu
} 
ences the difficulty of computing images. We use for our analysis the rendering equation as the integral of the product of functions and suggest that a meaningful characterization for classification of surface reflectance is its normalized variance defined carefully for sampling $\frac{1}{\pi \cos \theta}$ distribution. We advocate to use standard statistical tools instead of ad hoc approaches when such a characterization is needed in rendering algorithms.

\section{Motivation}

Our motivation came upon implementation of some algorithms such as photon mapping [Jensen 2001] that distinguishes between several types of light transport to deal with them efficiently based on the surface reflection. The question is whether to store photons on the surfaces that are 'almost diffuse'. A similar problem is whether to store virtual point light sources in the context of many-lights methods [Dachsbacher et al. 2013]. The algorithm needs a classification to distinguish which surfaces are 'diffuse or almost diffuse' to allow to store photons on these surfaces. [Heckbert 1990] provides the classification of the surface reflectance into diffuse, mirror and everything else as glossy, with possible separation of BRDF into its diffuse and glossy parts. He also provides the description of glossy part as the one that is inside the cone in the direction of ideal reflection. In practice the problem is not that simple, when we deal with real-world measured data. There are some options how to solve the problem. First, we can use methods such as independent component analysis to factor the BRDF into two parts and use diffuse part and the rest directly. This is costly and it also does not give any answer about how much diffuse is the surface reflectance. There are attempts for the answer, such as the measure of diffuseness $r_{d}=\rho_{\text {diffuse }} /\left(\rho_{\text {diffuse }}+\rho_{\text {specular }}\right)$ by [Balling and Marciniak 2009]. Second, we can fit the data to a chosen analytical BRDF model and use for our discrimination the parameters of the model such as the often used exponent or specular roughness for a particular BRDF model.

In general, fitting the data to a single BRDF model and using its selected parameter as some characterization is not possible for several reasons. First, some measured data including anisotropic ones or BTF, SVBRDF data can have several modalities, that can come also from spatial filtering. The multi-modality can be captured by some multi-lobe BRDF models such as [Lafortune et al. 1997] or in the context of bidirectional texture functions by a mixture of functions as in the model by [Wu et al. 2011]. Second, it is unclear how to put together the specular roughness from individual lobes. Third, even for a unimodal shaped distribution such as physically corrected Phong model [Lafortune and Willems 1994] it is unclear how to deal with it, since for a single lobe there is its shape given by exponent and specular albedo and diffuse albedo. Some analytical BRDF models can be fitted better to some measured data than the others [Ngan et al. 2005]. Some analytical models do not have explicit diffuse parts, for some BRDF models fitting is prohibitively expensive to be used in rendering algorithms. In practice (such as [Colbert et al. 2010]) it is common to restrict the number of BRDF models used to a few and then use some thresholds in algorithms that work acceptably for the selected BRDF models.

The design of such ad hoc approaches is well motivated but not theoretically backed up or driven by well justified objectives. We show in our analysis the solution when the objective is the minimization of the variance of rendering equation estimator.

\section{Related work}

There are many connections between our research and the former literature from different fields, due to the lack of space we limit our discussion to only a fraction of all possible sources.

\subsection{Appearance and perception}

The problem how much glossy the material looks like has been dealt with from the viewpoint of perception mostly by Hunter since the first work by Pfund in 1930, a more detailed survey for this topic is available in [Vangorp 2009]. There is a physical gloss measured by special gantries called glossmeters and there are tens of definitions how the gloss should be measured in dependence on the industry and application scenario. Further, Hunter already in 1937 presented that there are several different glosses: specular gloss, contrast gloss, sheen, absence-of-bloom gloss or haze, distinctnessof-image (DOI) gloss, surface-uniformity gloss. There are also other terms in use such as effulgence, shininess, burnish, luster that are difficult to be translated into other languages than English and they are rather vague. In computer graphics more recently [Pellacini et al. 2000] introduces a new BRDF model that is motivated by gloss reception. [Matusik et al. 2003] provides analysis of gloss space for 100 measured isotropic materials. More recent advances and citations to older work are surveyed in the theses of [Vangorp 2009] and [Leloup 2012].

\subsection{Image statistics, BRDF measurements, optics}

[Dror et al. 2001] use image statistics to estimate BRDF from images under natural illumination and show that it is possible to use variance, skew, kurtosis to estimate specular roughness of Ward BRDF model. This is then improved by [Ghosh et al. 2009] by the use of orthogonal spherical basis, where specular roughness of Ward model corresponds to normalization and scaling of standard deviation. They also demonstrate the use of this procedure on Ashikmin model. There is also a concept of surface roughness used in optics (for example by [Hoover and Gamiz 2006]) as $\sigma=\sigma_{h} / \lambda$, where $\sigma_{h}$ is the standard deviation of the surface height distribution and $\lambda$ is the illumination wavelength.

\subsection{Importance sampling in rendering algorithms}

The above surveyed work does not give any solution to optimize rendering algorithms. In addition to the above mentioned notation by [Heckbert 1990] Veach and Guibas [Veach and Guibas 1995] and Veach in his thesis mention the concept of specular roughness when developing multiple importance sampling (MIS), we quote [Veach 1997, page 253]:

“ In particular, we consider spherical light sources of varying radii, and glossy materials that have a surface roughness parameter $(r)$ that determines how sharp or fuzzy the reflections are. Smooth surfaces $(r=0)$ correspond to highly polished, mirror-like reflections, while rough surfaces $(r=1)$ correspond to diffuse reflection. It is possible to simulate a variety of surface finishes by using intermediate roughness values in the range $0<r<1$."

Then the roughness is used in Phong model as exponent $\alpha=$ $1 / r-1$, and this is used to report the results for testing MIS. [Pajot et al. 2011] present a concept of 'representativity' to improve 
on the robustness of estimators when using MIS in numerical integration. They observed the cases when the sampling leads to high variance and propose an empirical measure to deal with the problem. From the two representativity measures presented in the paper the empirical measure based on BRDF is called 'directionality' and consists of three parts, diffuse $d_{d}$, specular $d_{s}$, and mirror-like $d_{t}$. They give the derivation of directionality for physical Phong model. For diffuse part $d_{d}=1 /(2 . \pi)$, for glossy part of roughness of physical Phong model as $d_{s}=1 / 2 \pi+(1-1 / 2 \pi)(\pi / 3-$ $\left.\cos ^{-1}\left((1 / 2)^{\frac{1}{n+\alpha}}\right)\right) \cdot 3 / \pi$. For mirror-like interaction $d_{t}=1$. The three terms are composed by albedo for the three parts to a single term $R\left(f_{r}\left(x, \omega_{i}, \omega_{o}\right)\right)$ that is zero for diffuse BRDF and one for mirror-like surface. This proposal on representativity is not theoretically backed up and cannot be generalized to arbitrary surface reflectance.

A well-known representative of consistent algorithms that need surface reflectance characterization is photon mapping by Jensen [2001], where photons of the global photon map should be stored on "mostly diffuse surfaces". More recently, the need for concept to quantify either diffuseness or glossiness to drive the computation is used by Dammertz et al. [2010]. They deal with the similar concept as Veach, given characterization $r=0$ for diffuse and $r=1$ for specular for exponent of physically based model. They use however proportional formulation in the form of physical Phong model the exponent $\alpha=1024 . r$ and threshold $r_{T}=0.2$, but this is also ad hoc formula not theoretically founded.

The practitioners deal with the problem of surface reflectance characterization by using empirical functions derived from running the computation and using the tested values or ad hoc formulas for different algorithms and BRDF models. Even for a simple case such as environment map illumination it is needed and shown to be efficient by Colbert et al. [2010]. Their proposed solution is valid only for a single preselected BRDF model restricting parameters to a one or two and a single environment map.

\section{BRDF properties}

Bidirectional reflectance distribution function (BRDF) is defined by [Nicodemus et al. 1977] by means of the reflectance equation:

$$
\begin{aligned}
f_{r}\left(x, \omega_{o}, \omega_{i}\right) & =\frac{d L\left(x \rightarrow \omega_{o}\right)}{L\left(x \leftarrow \omega_{i}\right) \cdot \cos \left(\theta_{i}\right) d \omega_{i}} \\
& =\frac{d L\left(x \rightarrow \omega_{o}\right)}{L\left(x \leftarrow \omega_{i}\right) \cdot\left(\omega_{i} \cdot \vec{n}\right) d \omega_{i}}
\end{aligned}
$$

In this definition it fulfills Helmholtz reciprocity given as

$$
f_{r}\left(x, \omega_{o}, \omega_{i}\right)=f_{r}\left(x, \omega_{i}, \omega_{o}\right) .
$$

Observe that $f_{r}\left(x, \omega_{o}, \omega_{i}\right)$ is not in principle a probability density function (as it does not integrate to one with incoming directions $\omega_{i}$, but see below), it is unit-less (i.e. unit is $\left[\mathrm{sr}^{-1}\right]$, but this is still unit-less as steradian is unit-less). To obtain a probability density function (pdf) from the BRDF, it should be redefined using the reflectance equation. We can define it in the following way, for fixed $\omega_{o}$ and integrating over incoming directions $\omega_{i}$ :

$$
\operatorname{pdf}\left(x, \omega_{o}, \omega_{i}\right)=\frac{1}{a\left(x, \omega_{o}\right)} \cdot f_{r}\left(x, \omega_{i}, \omega_{o}\right) \cdot\left(\omega_{i} \cdot \vec{n}\right),
$$

where albedo $a\left(x, \omega_{o}\right)$ for fixed outgoing direction $\omega_{o}$ is defined as:

$$
a\left(x, \omega_{o}\right)=\int_{\Omega} f_{r}\left(x, \omega_{i}, \omega_{o}\right) \cdot\left(\omega_{i} \cdot \vec{n}\right) d \omega_{i}
$$

And then:

$\int_{\Omega} \operatorname{pdf}\left(x, \omega_{o}, \omega_{i}\right) d \omega_{i}=\frac{1}{a\left(x, \omega_{o}\right)} \int_{\Omega} f_{r}\left(x, \omega_{i}, \omega_{o}\right) \cdot\left(\omega_{i} \cdot \vec{n}\right) d \omega_{i}=1$

Observe that albedo $a\left(x, \omega_{o}\right)$ can be considered as $\pi$ times the expected value of $f_{r}\left(x, \omega_{i}, \omega_{o}\right)$ when sampling with pdf $\left(\omega_{i} \cdot \vec{n}\right) \cdot \frac{1}{\pi}$ :

$a\left(x, \omega_{o}\right)=\pi \int_{\Omega} f_{r}\left(x, \omega_{i}, \omega_{o}\right) \cdot \frac{1}{\pi}\left(\omega_{i} \cdot \vec{n}\right) d \omega_{i}=\pi E\left[f_{r}\left(x, \omega_{i}, \omega_{o}\right)\right]$

What does not have physical meaning is using as pdf the BRDF $f_{r}\left(x, \omega_{i}, \omega_{o}\right)$ with uniform density over $\omega_{i}$, although it would be indeed a pdf conveniently normalized:

$$
\int_{\Omega} \operatorname{pdf}_{2}\left(x, \omega_{o}, \omega_{i}\right) d \omega_{i}=\frac{1}{a^{\prime}\left(x, \omega_{o}\right)} \int_{\Omega} f_{r}\left(x, \omega_{i}, \omega_{o}\right) d \omega_{i}=1,
$$

where $a^{\prime}\left(x, \omega_{o}\right)$ is computed as:

$$
a^{\prime}\left(x, \omega_{o}\right)=\int_{\Omega} f_{r}\left(x, \omega_{i}, \omega_{o}\right) d \omega_{i}
$$

\section{Importance Sampling in Rendering Equa- tion}

The rendering equation [Kajiya 1986] expresses the radiance with zero self-emission from a surface with normal vector $\vec{n}$ as:

$$
L\left(x, \omega_{o}\right)=\int_{\Omega} L\left(x, \omega_{i}\right) f_{r}\left(x, \omega_{i}, \omega_{o}\right) \cdot\left(\omega_{i} \cdot \vec{n}\right) d \omega_{i},
$$

where $L\left(x, \omega_{i}\right)$ is the incoming radiance in direction $\omega_{i}$. Suppose you want to solve this equation by Monte Carlo. Let us consider different possibilities. First, naive computation would be to uniform sampling $\omega_{i}$. Estimator would be:

$$
L\left(x, \omega_{o}\right) \approx 2 \pi \frac{1}{N} \sum_{1}^{N} L\left(x, \omega_{i}\right) f_{r}\left(x, \omega_{i}, \omega_{o}\right) \cdot\left(\omega_{i} \cdot \vec{n}\right)
$$

Then you can do importance sampling. First possibility is to do importance sampling upon incoming radiance $L\left(x, \omega_{i}\right)$, then the estimator for radiance becomes:

$$
L\left(x, \omega_{o}\right) \approx L_{i}^{a v e}(x) \frac{1}{N} \sum_{1}^{N} f_{r}\left(x, \omega_{i}, \omega_{o}\right) \cdot\left(\omega_{i} \cdot \vec{n}\right),
$$

where

$$
L_{i}^{a v e}(x)=\int_{\Omega} L\left(x, \omega_{i}\right) d \omega_{i}
$$

According to the importance sampling definition, it comes natural to include the cosine term in the importance sampling pdf, however, this is in practice difficult, costly, or impossible. This second estimator would be then:

$$
L\left(x, \omega_{o}\right) \approx L_{i}^{\prime a v e}(x) \frac{1}{N} \sum_{1}^{N} f_{r}\left(x, \omega_{i}, \omega_{o}\right),
$$

where $L_{i}^{\prime a v e}(x)$ is the average illumination on $x$

$$
L_{i}^{\prime a v e}(x)=\int_{\Omega} L\left(x, \omega_{i}\right)\left(\omega_{i} \cdot \vec{n}\right) d \omega_{i}
$$

Observe the variance of the estimator eq. 13 becomes null when $f_{r}$ is constant (i.e. diffuse case) unlike the estimator eq. 11 for which variance will become non-zero. 
Doing importance sampling on $f_{r}$ times cosine, we end up in the estimator for radiance

$$
L\left(x, \omega_{o}\right) \approx a\left(x, \omega_{o}\right) \frac{1}{N} \sum_{1}^{N} L\left(x, \omega_{i}\right)
$$

This is the symmetrical case of estimator eq. 13, and variance becomes null when incoming radiance is constant.

\subsection{Analysis for constant environment map and ar- bitrary BRDF}

We consider further as an example illumination by environment map, where by symbol $R\left(\omega_{i}\right)$ we denote the radiant intensity of environment map at direction $\omega_{i}$ (in Watts/steradian). Considering $R\left(x, \omega_{i}\right)=$ const $=R$, outgoing radiance $L\left(x, \omega_{o}\right)$ becomes:

$$
L\left(x, \omega_{o}\right)=R \cdot \int_{\Omega} f_{r}\left(x, \omega_{i}, \omega_{o}\right) \cdot\left(\omega_{i} \cdot \vec{n}\right) d \omega_{i}=R \cdot a\left(x, \omega_{o}\right)
$$

Thus albedo is the outgoing radiance for constant incoming radiance $R=1$. The estimator that includes the cosine term in the sampling, i.e. eq. 13 , becomes for $R=1$ :

$$
L\left(x, \omega_{o}\right) \approx \pi \frac{1}{N} \sum_{1}^{N} f_{r}\left(x, \omega_{i}, \omega_{o}\right)
$$

and for cosine term not included in sampling, i.e. eq. 11, the estimator is:

$$
L\left(x, \omega_{o}\right) \approx 2 \pi \frac{1}{N} \sum_{1}^{N} f_{r}\left(x, \omega_{i}, \omega_{o}\right) \cdot\left(\omega_{i} \cdot \vec{n}\right),
$$

as for eq. 14 we get $L_{i}^{a v e}=\pi$ and for eq. $12 L_{i}^{\prime a v e}=2 \pi$ for constant incoming radiance $R=1$. We can then estimate the variance $V\left(L\left(x, \omega_{o}\right)\right)$ of both Monte Carlo estimators eq. 17 and eq. 18 and hence the noise in the image. Observe that only with estimator 17 we can have (trivially) null variance, for the case $f_{r}\left(x, \omega_{i}, \omega_{o}\right)=$ const., i.e., in the diffuse or Lambertian case.

\subsection{Analysis for non-constant environment map and arbitrary BRDF}

If environment map is not constant, $L\left(x, \omega_{o}\right)$ along a primary ray for the point on an object illuminated by the environment map can be naively computed using eq. 10 as:

$$
L\left(x, \omega_{o}\right) \approx 2 \pi \frac{1}{N} \sum_{1}^{N} R\left(\omega_{i}\right) f_{r}\left(x, \omega_{i}, \omega_{o}\right) \cdot\left(\omega_{i} \cdot \vec{n}\right),
$$

where $\vec{n}$ is the normal of a surface for the point $x$. Here we consider full visibility to simplify the analysis.

We can resource to the two importance sampling estimators defined above, eq. 11, 15. Let us call them $L_{1}$ and $L_{2}$. They correspond to the expected value $E\left[R^{\text {ave }} f_{r}\left(x, \omega_{i}, \omega_{o}\right)\left(\omega_{i} \cdot \vec{n}\right)\right]$ when using as pdf $R\left(\omega_{i}\right) / R^{\text {ave }}$ and to the expected value $E\left[a\left(x, \omega_{o}\right) R\left(\omega_{i}\right)\right]$ when using as pdf $f_{r}\left(x, \omega_{i}, \omega_{o}\right) .\left(\omega_{i} \cdot \vec{n}\right) / a\left(x, \omega_{o}\right)$, where $R^{a v e}$ is equal to:

$$
R^{a v e}=\int_{\Omega} R\left(\omega_{i}\right) d \omega
$$

The two estimators become then:

$$
\begin{aligned}
& L_{1}\left(x, \omega_{o}\right)=R^{a v e} \frac{1}{N} \sum_{1}^{N} f_{r}\left(x, \omega_{i}, \omega_{o}\right)\left(\omega_{i} \cdot \vec{n}\right) \\
& L_{2}\left(x, \omega_{o}\right)=a\left(x, \omega_{o}\right) \frac{1}{N} \sum_{1}^{N} R\left(\omega_{i}\right)
\end{aligned}
$$

The variance of $V_{1}=V\left[R^{a v e} f_{r}\left(x, \omega_{i}, \omega_{o}\right)\left(\omega_{i} \cdot \vec{n}\right)\right]$ and $V_{2}=V\left[a\left(x, \omega_{o}\right) R\left(\omega_{i}\right)\right]$, in general the variance of $g(x)$, can be estimated by standard formula:

$$
\widehat{V[g(X)}]=\frac{\sum_{i=1}^{N}\left(g\left(x_{i}\right)-\left(\sum_{i=1}^{N} g\left(x_{i}\right)\right) / N\right)^{2}}{N-1}
$$

assuming known relation for multiplication of a function:

$$
V[s g(X)]=s^{2} V[g(X)]
$$

Using the equation to compute the variance of a product of two functions $h_{1}(x)$ and $h_{2}(x)$ so $g(x)=h_{1}(x) h_{2}(x)$, one of them corresponding to $f_{r}\left(x, \omega_{i}, \omega_{o}\right) .\left(\omega_{i} \cdot \vec{n}\right)$ and the second one to $R\left(\omega_{i}\right)$, so when sampling according to $h_{2}(x)$ we get the variance as follows:

$$
\begin{aligned}
s & =\int_{\Omega} h_{2}(x) d x \\
V\left[s h_{1}(X)\right] & =s^{2}\left(\int_{\Omega} h_{1}(x)^{2}\left(h_{2}(x) / s\right) d x-\left(E\left[h_{1}(X)\right]\right)^{2}\right) \\
& =s \int_{\Omega} h_{1}(x)^{2} h_{2}(x) d x-I^{2}=V[I]
\end{aligned}
$$

we can compute their exact values:

$V_{1}=R^{\text {ave }} \int f_{r}\left(x, \omega_{i}, \omega_{o}\right)^{2}\left(\omega_{i} \cdot \vec{n}\right)^{2} R\left(\omega_{i}\right) d \omega-L\left(x, \omega_{o}\right)^{2}$

$V_{2}=a\left(x, \omega_{o}\right) \int R\left(\omega_{i}\right)^{2} f_{r}\left(x, \omega_{i}, \omega_{o}\right) \cdot\left(\omega_{i} \cdot \vec{n}\right) d \omega-L\left(x, \omega_{o}\right)^{2}$

Suppose now we want to use $N$ samples distributed among both estimators, $n_{1}+n_{2}=N$. Any convex combination of $L_{1}$ and $L_{2}, \alpha L_{1}+(1-\alpha) L_{2}$ is an unbiased estimator of $L\left(x, \omega_{o}\right)$, i.e., $E\left[\alpha_{1} L_{1}+\alpha_{2} L_{2}\right]=\alpha E\left[L_{1}\right]+(1-\alpha) E\left[L_{2}\right]=L\left(x, \omega_{o}\right)$, as $E\left[L_{1}\right]=$ $E\left[L_{2}\right]=L\left(x, \omega_{o}\right)$. Its variance is equal to:

$$
\begin{aligned}
V\left[\alpha_{1} L_{1}+\alpha_{2} L_{2}\right] & =\alpha^{2} V\left[L_{1}\right]+(1-\alpha)^{2} V\left[L_{2}\right] \\
& =\frac{\alpha^{2}}{n_{1}} V_{1}+\frac{(1-\alpha)^{2}}{n_{2}} V_{2}
\end{aligned}
$$

\subsection{Multiple importance sampling variance}

The multiple importance sampling (MIS [Veach and Guibas 1995]) estimator for the studied problem in previous section can be written as a standard Monte Carlo estimator, where the pdf $f(x)$ is given by a mixture of distributions (see Veach's thesis [Veach 1997, section 9.2.2.1]), for considering two distributions we have:

$$
f(x)=\alpha_{1} h_{1}(x)+\alpha_{2} h_{2}(x), \quad \alpha_{1}+\alpha_{2}=1
$$

Using the derivation in [Havran and Sbert 2014] we can formulate the expected value of $\frac{h_{1}(x) h_{2}(x)}{f(x)}$ when samples are taken according to pdf $f(x)$ as follows: $\mu=\int \frac{h_{1}(x) h_{2}(x)}{f(x)} f(x) d x=$ $\int \frac{h_{1}(x) h_{2}(x)}{f(x)}\left(\alpha_{1} h_{1}(x)+\alpha_{2} h_{2}(x)\right) d x=\alpha_{1} \mu_{1}+\alpha_{2} \mu_{2}$, and for both sampling strategies indexed by $k \in\{1,2\}$ we have $\mu_{k}=$ 
$\int \frac{h_{1}(x) h_{2}(x)}{f(x)} h_{k}(x) d x=E_{k}\left[\frac{h_{1}(x) h_{2}(x)}{f(x)}\right]$ the expected value of $\frac{h_{1}(x) h_{2}(x)}{f(x)}$ when samples are taken according to $h_{k}(x)$. The variance of $\frac{h_{1}(x) h_{2}(x)}{f(x)}$ can be computed as:

$$
\begin{aligned}
V\left[\frac{h_{1}(x) h_{2}(x)}{f(x)}\right] & =\int\left(\frac{h_{1}(x) h_{2}(x)}{f(x)}\right)^{2}\left(\sum_{k=1}^{2} \alpha_{k} h_{k}(x)\right) d x-\mu^{2} \\
& =\sum_{k=1}^{2} \alpha_{k} \int\left(\frac{h_{1}(x) h_{2}(x)}{f(x)}\right)^{2} h_{k}(x) d x-\mu^{2} \\
& =\sum_{k=1}^{2} \alpha_{k}\left(V_{k}\left[\frac{h_{1}(x) h_{2}(x)}{f(x)}\right]+\mu_{k}^{2}\right)-\mu^{2} \\
& =\sum_{k=1}^{2} \alpha_{k}\left(V_{k}\left[\frac{h_{1}(x) h_{2}(x)}{f(x)}\right]+\left(\mu_{k}-\mu\right)^{2}\right),(30)
\end{aligned}
$$

where $V_{k}\left[\frac{h_{1}(x) h_{2}(x)}{f(x)}\right]$ is the variance of $\frac{h_{1}(x) h_{2}(x)}{f(x)}$ with respect to function $h_{k}(x)$. Last equality comes from: $\sum \alpha_{k}\left(-2 \mu_{k} \mu+\mu^{2}\right)=$ $-2 \mu \sum \alpha_{k} \mu_{k}+\mu^{2}=-\mu^{2}$. More details for this derivation are in [Havran and Sbert 2014]. The interesting observation for us is that the variance of the result computed by MIS is the weighted sum of some variances (plus another term), similarly to the previous section.

\section{Proposal of BRDF characterization}

In rendering algorithms we aim to get the lowest variance of the estimator derived from the rendering equation such as those shown above. We have analyzed the variance $V$ of the product of functions $f(x)=h_{1}(x) h_{2}(x)$ as used in rendering equation, we have seen that variances of these functions $V\left[h_{1}(x)\right]$ and $V\left[h_{2}(x)\right]$ play central role in value of $V$. We have shown in Section 5.2 and 5.3 that the variance of the rendering equation is a weighted sum of variances of individual components for the linear combination of estimators and we have analyzed the variance of a product of two functions when using MIS.

As the rendering equation eq. 9 can be seen as the integral of a product of two functions $h_{1}(x) \cdot h_{2}(x)$ for $h_{1}(x)=f_{r}\left(x, \omega_{i}, \omega_{o}\right) \cdot\left(\omega_{i} \cdot \vec{n}\right)$ and $h_{2}(x)=R\left(\omega_{i}\right)$, we can study its limiting cases, when environment map $R(\omega)$ approaches a constant function. The variance $V\left[L\left(x, \omega_{o}\right)\right]$ when sampling from $h_{2}(x)$ in eq. 9 , for a slowly varying environment map $R\left(\omega_{i}\right)$, approaches the variance of BRDF function, being equal in the limit when $R(\omega)$ is constant, i.e., $V\left[L\left(x, \omega_{o}\right)\right]=V\left[f_{r}\left(x, \omega_{i}, \omega_{o}\right)\right]$ for sampling according to $1 / \pi\left(\omega_{i} \cdot \vec{n}\right)$. In the limit, when environment map becomes constant, the variance of the studied function $V\left[L\left(x, \omega_{o}\right)\right]$ is determined by the variance of surface reflectance $V\left[f_{r}\left(x, \omega_{o}\right)\right]$, for both eqs. 28 and 30 .

Clearly, the variance of $V\left[f_{r}\left(x, \omega_{o}\right)\right]$ plays a key role in for this simplified analysis, as almost always, when $f_{r}\left(x, \omega_{o}\right) \cdot \omega_{i} \cdot \vec{n}$ and $R\left(x, \omega_{o}\right)$ will not get correlated, the higher the value of $V\left[f_{r}\left(x, \omega_{o}\right)\right]$ the higher the value of $V\left[L\left(x, \omega_{o}\right)\right]$. Therefore we suggest to use the variance of BRDF in addition to another commonly used statistical characterization known as albedo $a\left(x, \omega_{o}\right)$ that as we have seen corresponds to $\pi$ times mean value $E\left[f_{r}\left(x, \omega_{o}\right)\right]$ in eq. 6 . We argue that this is more natural than using ad hoc definitions of diffuseness as used by some papers in the past and that our definition is enough general and not dependent on a particular BRDF model.

To make the proposed variance characterization approximately comparable in the perceptual domain for a pair or set of BRDFs, we suggest to use its normalized variant, known in statistics as in
Squared-Coefficient-of-Variation (e.g. in [Rubinstein and Kroese 2008], abbreviated as SCV). This then to some extent allows to visually compare perceived gloss if two BRDFs are normalized by albedo. For short, we suggest to use the term variant index of glossiness (abbreviated as VIG) defined as:

$$
\begin{aligned}
r_{g}\left(f_{r}\left(x, \omega_{o}\right)\right) & =\frac{V\left[f_{r}\left(x, \omega_{o}\right)\right]}{E^{2}\left[f_{r}\left(x, \omega_{o}\right)\right]}=\frac{E\left[\left(f_{r}\left(x, \omega_{o}\right)-E\left[f_{r}\left(x, \omega_{o}\right)\right]\right)^{2}\right]}{E^{2}\left[f_{r}\left(x, \omega_{o}\right)\right]} \\
& =\frac{\int\left(f_{r}\left(x, \omega_{o}\right)-\int f_{r}\left(x, \omega_{o}\right) \cdot\left(\omega_{i} \cdot \vec{n}\right) d \omega\right)^{2} \cdot\left(\omega_{i} \cdot \vec{n}\right) d \omega_{i}}{\left(\int f_{r}\left(x, \omega_{o}\right) \cdot\left(\omega_{i} \cdot \vec{n}\right) d \omega\right)^{2}},
\end{aligned}
$$

where variance $V\left[f_{r}\left(x, \omega_{o}\right)\right]$ and mean $E\left[f_{r}\left(x, \omega_{o}\right)\right]$ are computed according to $1 / \pi\left(\omega_{i} \cdot \vec{n}\right)$ distribution. Its properties are then so that for diffuse BRDF $r_{g}=0$, for mirror $r_{g}=\infty$. Since we know also albedo $a\left(x, \omega_{o}\right)=\pi E\left[f_{r}\left(x, \omega_{o}\right)\right]$, the non-normalized variance $V\left[f_{r}\left(x, \omega_{o}\right)\right]$ can be easily computed when needed from $r_{g}\left(f_{r}\left(x, \omega_{o}\right)\right)$ and albedo using eq. 6 . The normalization would correspond in rendering to equalizing brightness of images.

We expect that the statistics above can be computed in closed form for analytical BRDF models as we show in appendix on an example. For measured tabulated data it is possible to compute both variant index of glossiness and albedo numerically in precomputation and store them to a 1D (isotropic BRDF) or 2D (anisotropic BRDF) table similar to BRDF data, as this table has two dimensions less than the data the overhead storage is negligible. The values for different $\omega_{o}$ can be then interpolated from the values in table.

Note that the same characterization is computable for other used surface reflectance models, where Helmholtz reciprocity does not hold, such as BTFs. After normalization $r_{g}\left(f_{r}\left(x, \omega_{o}\right)\right)$ must be computed numerically.

When the BRDF model is given by deterministic mixture of several BRDF models, both characterization can be computed from its components easily using the known formulas: $E\left[\sum \alpha_{k} \cdot g_{k}(X)\right]=$ $\sum \alpha_{k} \cdot E\left[g_{k}(X)\right]$ for mean values and $V\left[\sum \alpha_{k} \cdot g_{k}(X)\right]=\sum \alpha_{k}^{2} V\left[g_{k}(X)\right]$ for variances.

We show $r_{g}\left(f_{r}\left(x, \omega_{o}\right)\right)$ in four videos (available at web page: http://dcgi.fel.cvut.cz/ havran/REFLVIG/) for rendering images of two objects (sphere and Stanford Bunny) using MERL BRDF data [Matusik et al. 2003] illuminated by two environment maps (Grace Cathedral and St'Peters Basilica).

\section{Limitations}

For layered BRDF functions such as [Weidlich and Wilkie 2007], where one BRDF is perfectly specular (such as lacquered wood), the VIG $r_{g}\left(f_{r}\left(x, \omega_{o}\right)\right)$ has to be computed separately for layers since for variance mixing infinity to anything results in infinity. This corresponds to handling such BRDFs in rendering algorithms in practice. The perfect mirror reflections are handled by deterministically shooting a single ray in ideally reflected direction, while diffuse and glossy layers are solved stochastically.

\section{Conclusion}

We have proposed a novel and easily computable surface reflectance characterization VIG that can be utilized instead of ad hoc methods used to classify on reflection bounces in the past. Instead of using the characterization as a measure of 'diffuseness' in range from zero to one we show that for rendering algorithms it is much more meaningful the measure of 'glossiness' computed as the variance of BRDF in range from zero (diffuse) to infinity (mirror) normalized by albedo for the sake of rough perceptual comparisons. We would like to advocate the use of standard statistical tools for surface reflectance models in applications and in future research. 
For future work we would like to study the use of the statistical characterization in rendering algorithms as surveyed in this paper and the use of statistical characterizations for other pdfs such as environment map, scattering functions etc.

\section{Acknowledgement}

In addition to MERL BRDF database [Matusik et al. 2003] we would like to thank Paul Debevec for data of environment maps and the Stanford 3D Scanning Repository for Bunny model. This work has been partially funded by the Czech Science Foundation of the Czech Republic under research project No. GA14-19213S and by the Grant Agency of the Czech Technical University in Prague, grant No. SGS13/214/OHK3/3T/13, and on the Spanish side by grants TIN2013-47276-C6-1-R from the Spanish Government, 2014 SGR 1232 from the Catalan Government and National Natural Science Foundation of China (Nos. 61372190 and 61331018).

\section{Appendix A}

Analytical BRDF models are often designed to allow for importance sampling analytically. Some of them can have analytical form of their variance and hence the characterization index $r_{g}(X)$. We show here the formula for $r_{g}(X)$ (eq. 31) of physically based variant of Phong model presented by [Lafortune and Willems 1994]. We can derive the variance of the Lafortune-Phong model assuming the whole lobe is above the surface. The simplest case is when the outgoing direction is along the surface normal. We start with the formulation:

$$
f_{r}\left(\omega_{o}, \omega_{i}\right)=\frac{\rho_{d}}{\pi}+\rho_{s} \frac{n+2}{2 \pi} \cdot \cos ^{n}(\theta)
$$

and knowing $d \omega_{i}=\sin \theta d \theta d \phi$, we then solve two integrals:

$$
\begin{aligned}
& \pi E\left[X^{2}\right]=\int f_{r}^{2}\left(\omega_{o}, \omega_{i}\right) \cos \theta d \omega_{i} \\
& =\left(\frac{\rho_{d}}{\pi}+\rho_{s} \frac{n+2}{2 \pi} \cos ^{n}(\theta)\right)^{2} \cos \theta \sin \theta d \theta d \phi \\
& =\left(\frac{\rho_{d}}{\pi}\right)^{2} \cos \theta \sin \theta d \theta d \phi \\
& +2 \frac{\rho_{d}}{\pi} \rho_{s} \frac{n+2}{2 \pi} \cos ^{n+1}(\theta) \sin \theta d \theta d \phi \\
& +\left(\rho_{s} \frac{n+2}{2 \pi}\right)^{2} \cos ^{2 n+1}(\theta) \sin \theta d \theta d \phi \\
& =-\frac{1}{2}\left(\frac{\rho_{d}}{\pi}\right)^{2}\left[\cos ^{2} \theta\right]_{0}^{\pi / 2} 2 \pi \\
& -\frac{1}{n+2} 2 \frac{\rho_{d}}{\pi} \rho_{s} \frac{n+2}{2 \pi}\left[\cos ^{n+2}(\theta)\right]_{0}^{\pi / 2} 2 \pi \\
& -\frac{1}{2 n+2}\left(\rho_{s} \frac{n+2}{2 \pi}\right)^{2}\left[\cos ^{2 n+2}(\theta)\right]_{0}^{\pi / 2} 2 \pi \\
& =\frac{1}{2}\left(\frac{\rho_{d}}{\pi}\right)^{2} 2 \pi \\
& +\frac{1}{n+2} 2 \frac{\rho_{d}}{\pi} \rho_{s} \frac{n+2}{2 \pi} 2 \pi \\
& +\frac{1}{2 n+2}\left(\rho_{s} \frac{n+2}{2 \pi}\right)^{2} 2 \pi \\
& =\frac{\rho_{d}^{2}}{\pi}+\frac{2 \rho_{d} \rho_{s}}{\pi}+\rho_{s}^{2} \frac{(n+2)^{2}}{(2 n+2) 2 \pi}
\end{aligned}
$$

The expected value of the BRDF model given in [Lafortune and Willems 1994] we give here for the sake of completeness as it was designed to correspond to albedo:

$$
\begin{aligned}
\pi E[X]= & \int f_{r}\left(\omega_{o}, \omega_{i}\right) \cos \theta d \omega_{i} \\
= & \left(\frac{\rho_{d}}{\pi}+\rho_{s} \frac{n+2}{2 \pi} \cos ^{n}(\theta)\right) \cos \theta \sin \theta d \theta d \phi \\
= & \frac{\rho_{d}}{\pi} \cos \theta \sin \theta d \theta d \phi \\
& +\rho_{s} \frac{n+2}{2 \pi} \cos ^{n+1}(\theta) \sin \theta d \theta d \phi \\
= & -\frac{1}{2} \frac{\rho_{d}}{\pi}\left[\cos ^{2} \theta\right]_{0}^{\pi / 2} 2 \pi \\
& -\frac{1}{n+2} \rho_{s} \frac{n+2}{2 \pi}\left[\cos ^{n+2}(\theta)\right]_{0}^{\pi / 2} 2 \pi \\
= & \frac{1}{2} \frac{\rho_{d}}{\pi} 2 \pi+\frac{1}{n+2} \rho_{s} \frac{n+2}{2 \pi} 2 \pi \\
= & \rho_{d}+\rho_{s}
\end{aligned}
$$

Then variant index of glossiness (VIG) $r_{g}(X)$ becomes:

$$
\begin{aligned}
r_{g}(X) & =V\left[f_{r}\left(x, \omega_{o}\right)\right] / E^{2}\left[f_{r}\left(x, \omega_{o}\right)\right] \\
& =\frac{\rho_{d}^{2}+2 \rho_{d} \cdot \rho_{s}+\rho_{s}^{2} \frac{(n+2)^{2}}{(2 n+2) 2}-\left(\rho_{d}+\rho_{s}\right)^{2}}{\left(\rho_{d}+\rho_{s}\right)^{2}} \\
& =\frac{\rho_{s}^{2}\left(\frac{(n+2)^{2}}{(2 n+2) 2}-1\right)}{\left(\rho_{d}+\rho_{s}\right)^{2}}
\end{aligned}
$$

\section{Appendix B}

We reproduce here the images from [Havran and Sbert 2014] that shows the visualization of variance for spatially varying LafortunePhong BRDF model [Lafortune and Willems 1994] used above in Figure 2 with rainbow pseudocolor mapping between blue (lowest variance) and red (highest variance), when diffuse albedo in $y$ axis changes linearly and the specular exponent $n$ in $x$ axis using this formula $n(x)=-1-0.111211 /\left(0.1 . x^{0.2}-0.101101\right)$ for $x \in\langle 0,1\rangle$ that gives range for $n$ as $\langle 0.1,100\rangle$, assuming the viewer direction is aligned with the surface normal.

In Figure 3 we give the 3D graphs of variant index of glossiness $r_{g}\left(f_{r}\left(x, \omega_{o}\right)\right)$ for different values of $\rho_{d}$ and specular index $n$ for the same Lafortune-Phong BRDF model, so visualization of eq. 33 .

\section{References}

Balling, B. L., And Marciniak, M. A. 2009. Comparison of Several Surfaces for Use as a MWIR BRDF Standard. In Proceedings of SPIE 7453, Infrared Spaceborne Remote Sensing and Instrumentation XVII, SPIE, 74530O-74530O-10.

Colbert, M., Premoze, S., And Francois, G. 2010. Importance Sampling for Production Rendering. In ACM SIGGRAPH 2010 Course Notes, ACM SIGGRAH.

Dachsbacher, C., KŘivánek, J., Hašan, M., Arbree, A., WALTER, B., AND NOVÁK, J. 2013. Scalable Realistic Rendering with Many-Light Methods. Computer Graphics Forum 32, 1. 


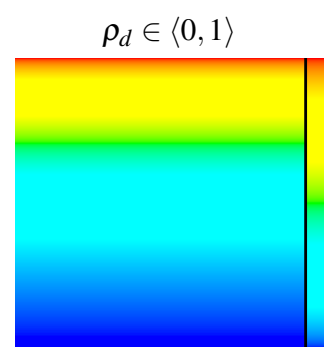

(a)

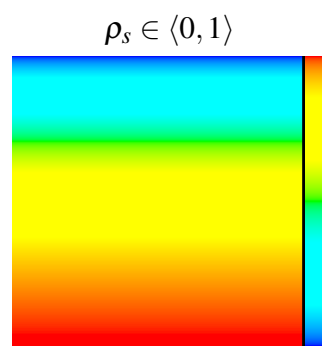

(b)

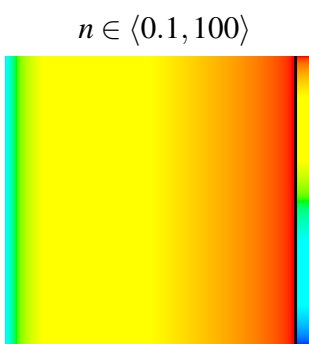

(c)

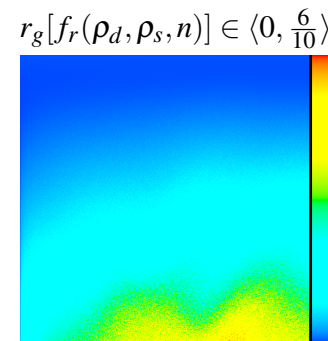

(d)

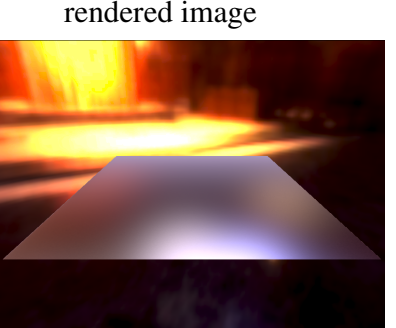

(e)

Figure 2: The visualization of $r_{g}(X)$ for Lafortune-Phong BRDF model $X=\rho_{d} / \pi+\rho_{s} \frac{n+2}{2 \pi} \cos ^{n}(\theta)$ using rainbow color mapping for spatially varying properties on 2D square. (a) diffuse albedo $\rho_{d}$, (b) specular albedo $\rho_{s}$ (albedo $\rho_{d}+\rho_{s}=1$ ), (c) specular exponent $n$ in range $\langle 0.1,100\rangle$, (d) variant index of glossiness $r_{g}(X)$ of the rectangle with spatially varying BRDF for 10,000 samples shown in range $\left\langle 0, \frac{6}{10}\right\rangle$ (corresponds to variance when sampled from constant environment map), (e) converged rendered image from Grace Cathedral environment map.

Dammertz, H., Keller, A., And Lensch, H. 2010. Progressive Point-Light-Based Global Illumination. Computer Graphics Forum 29, 8, 2504-2515.

Dror, R., Adelson, E., AND Willsky, A. 2001. Recognition of Surface Reflectance Properties from a Single Image under Unknown Real-World Illumination. In MIT AIM.

Ghosh, A., Chen, T., Peers, P., Wilson, C. A., And Debevec, P. 2009. Estimating Specular Roughness and Anisotropy from Second Order Spherical Gradient Illumination. In Proceedings of the Twentieth Eurographics conference on Rendering, Eurographics Association, Aire-la-Ville, Switzerland, Switzerland, EGSR'09, 1161-1170.

Havran, V., AND Sbert, M. 2014. Optimal Combination of Techniques in Multiple Importance Sampling. In Proceedings of the 13th ACM SIGGRAPH International Conference on VirtualReality Continuum and Its Applications in Industry, ACM, New York, NY, USA, VRCAI '14, 141-150.

Heckbert, P. S. 1990. Adaptive Radiosity Textures for Bidirectional Ray Tracing. In Proceedings of the 17th annual conference on Computer graphics and interactive techniques, ACM, New York, NY, USA, SIGGRAPH '90, 145-154.

Hoover, B., AND Gamiz, V. 2006. Coherence Solution for Bidirectional Reflectance Distributions of Surfaces with WavelengthScale Statistics. J Opt Soc Am A Opt Image Sci Vis 23, 2, 314-28.

Jensen, H. W. 2001. Realistic Image Synthesis Using Photon Mapping. A. K. Peters, Ltd., Natick, MA, USA.

KAJIYA, J. T. 1986. The Rendering Equation. In Computer Graphics (SIGGRAPH '86 Proceedings), D. C. Evans and R. J. Athay, Eds., vol. 20, 143-150.

Lafortune, E. P., And Willems, Y. D. 1994. Using the Modified Phong Reflectance Model for Physically Based Rendering. Tech. Rep. report CW197, Dept. of Computer Science, K.U.Leuven.

Lafortune, E. P. F., Foo, S.-C., Torrance, K. E., And GreEnBerG, D. P. 1997. Non-Linear Approximation of Reflectance Functions. In Proceedings of the 24th annual conference on Computer graphics and interactive techniques, ACM Press/Addison-Wesley Publishing Co., New York, NY, USA, SIGGRAPH '97, 117-126.

Leloup, F. 2012. New Methods and Models Improving the Prediction of Visual Gloss Perception. PhD thesis, Department of Computer Science, Faculty of Engineering Science, KU Leuven.
Matusik, W., Pfister, H., Brand, M., And McMillan, L. 2003. A Data-driven Reflectance Model. ACM Trans. Graph. 22, 3 (July), 759-769.

NGan, A., Durand, F., AND Matusik, W. 2005. Experimental Analysis of BRDF Models. In Proceedings of the Eurographics Symposium on Rendering, Eurographics Association, 117-226.

Nicodemus, F. E., Richmond, J. C., Hsia, J. J., GinsberG, I. W., AND LIMPERIS, T. 1977. Geometric Considerations and Nomenclature for Reflectance. Monograph 161, National Bureau of Standards (US), October.

Pajot, A., Barthe, L., Paulin, M., and Poulin, P. 2011. Representativity for Robust and Adaptive Multiple Importance Sampling. IEEE Transactions on Visualization and Computer Graphics 17, 8 (Aug.), 1108-1121.

Pellacini, F., Ferwerda, J. A., And Greenberg, D. P. 2000. Toward a Psychophysically-Based Light Reflection Model for Image Synthesis. In Proceedings of the 27th annual conference on Computer graphics and interactive techniques, ACM Press/Addison-Wesley Publishing Co., New York, NY, USA, SIGGRAPH '00, 55-64.

Rubinstein, R., And Kroese, D. 2008. Simulation and the Monte Carlo Method. Wiley Series in Probability and Statistics. Wiley.

VANGORP, P. 2009. Human Visual Perception of Materials in Realistic Computer Graphics. PhD thesis, Department of Computer Science, Faculty of Engineering Science, KU Leuven, Leuven, Belgium.

Veach, E., AND Guibas, L. J. 1995. Optimally Combining Sampling Techniques for Monte Carlo Rendering. In Proceedings of the 22nd annual conference on Computer graphics and interactive techniques, ACM, New York, NY, USA, SIGGRAPH '95, 419-428.

VEACH, E. 1997. Robust Monte Carlo Methods for Light Transport Simulation. PhD thesis, Stanford University.

WeIDLICH, A., AND WILKIE, A. 2007. Arbitrarily layered microfacet surfaces. In GRAPHITE 2007, ACM, 171-178.

Wu, H., Dorsey, J., AND Rushmeier, H. 2011. A Sparse Parametric Mixture Model for BTF Compression, Editing and Rendering. Computer Graphics Forum 30, 465-473. 

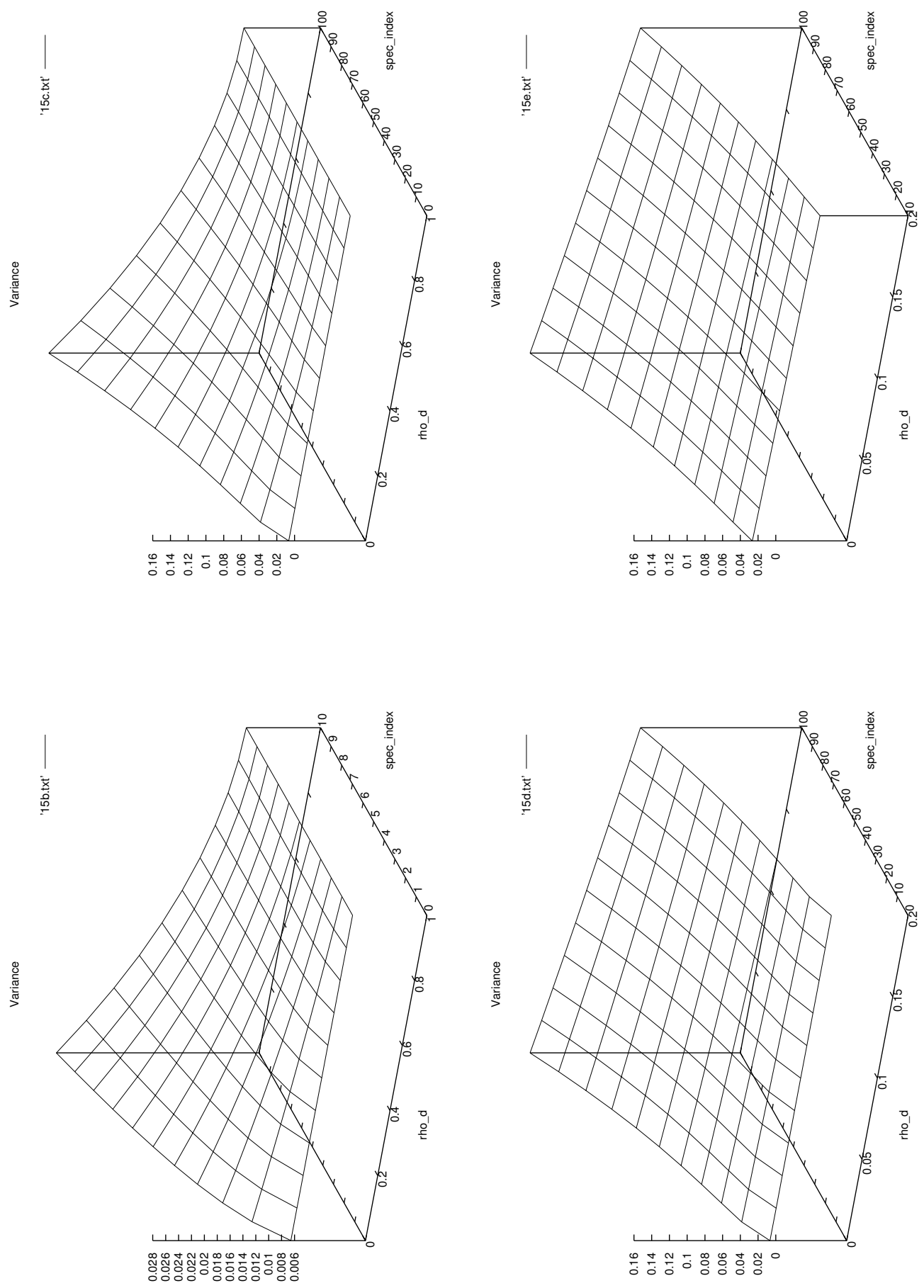

Figure 3: The variant index of glossiness (VIG) $r_{g}(X)$ for Lafortune-Phong model for unit albedo $\left(\rho_{d}+\rho_{s}=1\right)$ and range of values (top left) $\rho_{d}=0 \ldots 1, \rho_{s}=1-\rho_{d}$, and exponent $n=0 \ldots 100$. (top right) $\rho_{d}=0 \ldots \frac{1}{5}, \rho_{s}=1-\rho_{d}$, and exponent $n=0 \ldots 100$. (bottom left) $\rho_{d}=0 \ldots 1, \rho_{s}=1-\rho_{d}$, and exponent $n=0 \ldots 10$. (bottom right) $\rho_{d}=0 \ldots \frac{1}{5}, \rho_{s}=1-\rho_{d}$, and exponent $n=0 \ldots 100$. 